\title{
Eye Movement Patterns in Solving Science Ordering Problems
}

\author{
Hui Tang \\ University of Georgia \\ Lisa Kendhammer \\ University of Georgia \\ Scott A. Brown \\ University of Georgia
}

\author{
Elizabeth Day \\ University of Georgia \\ James N. Moore \\ University of Georgia \\ Norbert J. Pienta \\ University of Georgia
}

\begin{abstract}
Dynamic biological processes, such as intracellular signaling pathways, commonly are taught using static representations of individual steps in the pathway. As a result, students often memorize these steps for examination purposes, but fail to appreciate the cascade nature of the pathway. In this study, we compared eye movement patterns for students who correctly ordered the components of an important pathway responsible for vasoconstriction against those who did not. Similarly, we compared the patterns of students who learned the material using three dimensional animations previously associated with improved student understanding of this pathway against those who learned the material using static images extracted from those animations. For two of the three ordering problems, students with higher scores had shorter total fixation duration when ordering the components and spent less time (fixating) in the planning and solving phases of the problem-solving process. This finding was supported by the scanpath patterns that demonstrated that students who correctly solved the problems used more efficient problem-solving strategies.
\end{abstract}

Keywords: Eye-tracking, problem-solving, scanpath, science ordering problems

\section{Introduction}

It is common practice in the biological sciences for students to learn dynamic processes, such as biochemical cascades, using a series of static images or simple diagrams in textbooks in which components of a pathway are connected by arrows. Unfortunately, many students fail to fully understand either that the cascade itself is critical for the process to occur or the spatial relationships among the components of the cascade.

To address these problems, three dimensional (3-D) animations have been created that depict the dynamic nature of these processes (Buchanan et al, 2005; Reindl et al, 2015). In the former study, students' scores for content, and understanding both the cascade nature and

Received December 15, 2015; Published May 15, 2016.

Citation: Tang, H., Day, E., Kendhammer, L., Moore, J. N., Brown, S. A., \& Pienta, N. J. (2016). Eye movement patterns in solving science ordering problems. Journal of Eye Movement Research, 9(3):6, 1-3.

Digital Object Identifier: 10.16910/jemr.9.3.6

ISSN: $1995-8692$

This article is licensed under a Creative Commons Attribution 4.0 International license. (oc) $\mathrm{EY}$ spatial organization of the pathway were significantly higher when they were taught using the animations.

In order for students to envision how alterations in intracellular pathways might cause adverse effects, they first must be able to mentally reconstruct the pathway. There are two approaches to assess students' understanding of this material. One is to present the components in different orders, and task the students with recognizing the correct one. The limitation to this approach is that a student simply must identify one component in the incorrect position to eliminate that choice. The other approach is to present the components in random order and have the students rearrange them in the correct order. If combined with eye-tracking technology, this approach can provide opportunities for researchers to more accurately assess the students' series of steps of problem-solving.

\section{Eye-tracking and Problem-solving}

Eye-tracking research is based on the eye-mind hypothesis, which states that eye movement is the observable measure of visual attention that is linked to the cognitive processing of information (Just \& 
Carpenter, 1984; Eivazi \& Bednarik, 2011; Doherty, O'Brien \& Carl, 2010). The use of eye-tracking to study problem-solving in science and mathematics education has emerged as a viable technique in the last three decades (Suppes, Cohen, Laddaga, Anliker \& Floyd, 1983; De Corte, Verschaffel \& Pauwels, 1990; Hegarty, Mayer \& Green, 1992; Epelboim \& Suppes, 2001; Grant \& Spivey, 2003; Green, Lemaire \& Dufau, 2007; Tang \& Pienta, 2012; Tang, Kirk \& Pienta, 2014). The majority of these studies have utilized multiple-choice problems that can be solved by choosing the correct answer with a simple mouse click. The design and data analysis of such studies are uncomplicated, and the experimental procedure is straightforward and short. However, the simplistic nature of multiple-choice items requires the use of complementary experimental methods like verbal protocols to support the researchers' conclusions.

To obtain insight into participants' cognitive processes, researchers have utilized more complex formats of items including arithmetic equations, graphs, animations or simulations. In many of these studies, participants verbalized their solutions during eyetracking (concurrent "think aloud") or post-experiment interviews (retrospective "think aloud") (van Gog, Paas \& van Merriënboer, 2005). Nevertheless, transcribing and coding verbal data are time-consuming and costly (Chi, 1997; Holmqvist, Nyström, Andersson, Dewhurst, Jarodzka \& Van de Weijer, 2011, p. 292), and "think aloud" interviews may influence participants' behaviors and/or mental workload in certain settings (Hertzum, Hansen \& Andersen, 2009).

Therefore, a problem type that can reveal participants' cognitive processes with relatively simple eye-tracking data should overcome the above shortcomings. Ordering problems (also called sequencing or drag-and-drop problems) task students with placing a randomized set of items or steps into their correct order. This type of problem is ideal for investigations of the students' understanding of the interdependency of individual steps in a biological process, as was the focus of the present study. It is also ideal for an eye-tracking experiment, because participants can click and move the mouse to rearrange the items on a computer screen, thereby eliminating the need for participants to "think aloud" or write on computer screens while their step-wise approach to the solution (i.e., problem-solving strategy) is recorded.

In the present study, we conducted an eye-tracking experiment to investigate undergraduate students' eye movement patterns and corresponding cognitive activities as they solved ordering problems in physiology. To understand how students solve problems, some researchers divide problem-solving processes into sub-phases. It has been found that eye movement patterns in the same phases can be different between two groups of participants, or can vary in different phases within a group (Tang \& Pienta, 2012; Tang et al., 2014; Hegarty et al., 1992). Depending on the characteristics of the task being performed, the phases and their components have been proposed differently (Hegarty et al., 1992; De Corte et al., 1990; Green et al., 2007; Mayer, Larkin \& Kadane, 1984). In this study, we divided the problem-solving process into three phases: reading-and-planning, problem-solving and answer-checking. Reading-and-planning is defined from the time when a participant began to read a problem to the time before the participant completely moved a choice into a step box. Problem-solving is defined from the end of reading-and-planning to the time when the participant moved the last choice into a step box and did not make any subsequent changes. The third phase, answer-checking, is from the end of problem-solving to the time when the participant clicked the "submit" button.

\section{Eye Movement Measures}

Eye-tracking studies typically use two basic types of measures: fixation and saccade. Scanpath, a third type of eye movement measure, also is used (Poole \& Ball, 2006). A fixation is defined as "the state when the eye remains (still) over a period of time". A saccade is "the rapid motion of the eye from one fixation to another" (Holmqvist et al., 2011, p. 21-23). A scanpath is simply constructed from the fixations and saccades using temporal sequencing.

In eye-tracking research, fixation duration may be the most-frequently reported eye movement measure; other common measures include fixation count and visit count (Holmqvist et al., 2011, p. 377, 412-417). According to the Tobii Studio 2.X manual (Tobii, 2010), fixation duration measures "the duration of each individual fixation within an area of interest (AOI)"; fixation count "measures the number of times the participant fixates on an AOI or an AOI group"; and visit count "measures the number of visits within an AOI or AOI group".

It is generally accepted that fixation duration and fixation count are correlated to difficulty, complexity, 
importance and interest of visual materials or tasks, which reflect the cognitive load of the tasks (Slykhuis, Wiebe \& Annetta, 2005). Specifically, longer fixation duration corresponds to higher complexity of visual materials; larger fixation number indicates more importance of the region being perceived (Hegarty, Mayerm \& Green, 1992; Green, Lemaire \& Dufau, 2007). Furthermore, scanpath reflects viewing strategies, which imply processes of cognition (Slykhuis et al., 2005; Peebles \& Cheng, 2001). As a result, researchers use scanpath patterns to examine characteristics of one's cognitive process and to differentiate problem-solving strategies between groups of participants. Studies have shown that experts and novices have different scanpath patterns when they view materials and solve problems (Tai, Loehr \& Brigham, 2006; van Gog, et al., 2009a; Tang, Topczewski, Topczewski \& Pienta, 2012). For example, experts more quickly find and look relatively longer at relevant information, and thus solve problems more effectively than novices (Tang et al., 2012; Tsai et al., 2012).

\section{Purpose of Study}

The purpose of this study was to explore the differences in eye movement patterns between students who correctly ordered the components of the alpha-adrenergic pathway and those who did not. The authors also were interested in possible correlations between the eye movement measures themselves, as well as students' scanpath patterns during problem solving. Finally, the effects of media type (dynamic animations vs. static images) on student performance were examined. The research questions were:

1. What are the correlations between fixation duration, fixation count, visit count, time on task and number of mouse clicks?

2. What are the relationships between student performance and the above factors?

3. What are the differences in scanpath patterns between students who solved the problems correctly and incorrectly?

4. How does media type affect whether students solve the problems correctly or incorrectly?

\section{Methodology}

\section{Participants}

The participants were students enrolled in an undergraduate physiology course at a large Southeastern comprehensive university during the spring 2014 semester. A total of 89 students volunteered to participate in the Institutional Review Board (IRB) approved study and received compensation for their time. Approximately $80 \%$ of the participants had previously taken a biochemistry course that covered the alpha-receptor pathway used as the subject of this study. The participants were randomly assigned to two groups that differed by the type of educational media they viewed -- dynamic animations versus static images. Out of the 89 participants, data from eight were discarded due to poor quality of eyetracking recordings, which was based on the cutoff value $(80 \%)$ of the eye-tracking ratio (Kruger, Hefer \& Matthew, 2014). Consequently, 81 participants consisting of 31 male participants and 50 female participants remained in the analysis. Among the 81 participants, 39 watched the media containing dynamic animations and 42 watched the media containing static images.

\section{Apparatus}

Participants' eye movements were recorded using a Tobii T120 eye tracker with a sampling rate of $60 \mathrm{~Hz}$. The threshold for defining a fixation was set to be 100 ms with a radius of 30 pixels, with an accuracy of 0.5 degrees. The display of the eye tracker included a 17inch LCD screen with a resolution of $1280 \times 1024$ pixels. The data were recorded and processed using Tobii Studio 2.0.4 software (Tobii, 2010).

\section{Materials}

The educational media used in the experiment, which simulated the process of the alpha receptor pathway, was designed by the authors. The two types of media, dynamic animation and static images, had the same length ( $5 \mathrm{~min})$ and content, including the same narration. The animations were those used in the study by Buchanan et al. (2005) and depicted the dynamic interactions of the processes. The static images displayed static representations, which were 30 screenshots from the dynamic animations. Figure 1 shows a representative static image. 


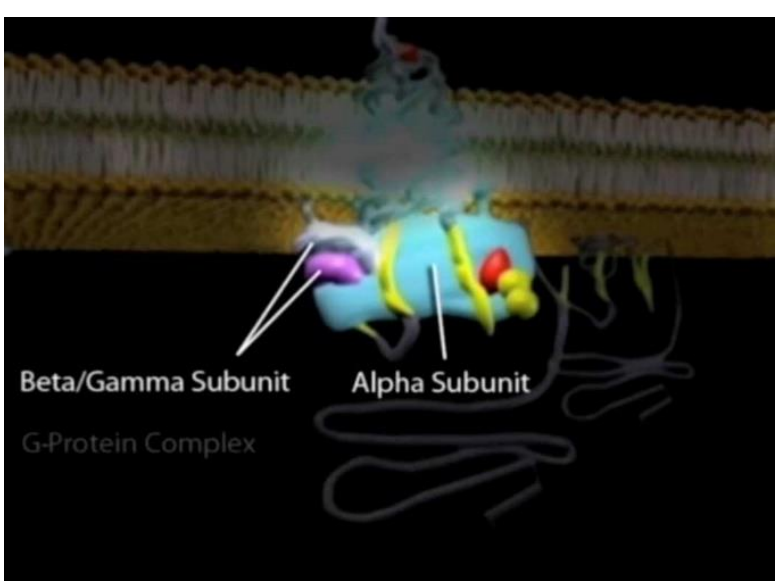

Figure 1. Screenshot of the media showing components of the alpha receptor pathway.

After watching the media, the participants were presented 17 problems to solve, which were in the same order. These problems were developed by the authors and were available on the intranet hosted by the same server where the eye tracker was connected. Each problem was displayed on a corresponding website with a submit button. Two types of problems were designed, one was multiple-choice (14 problems) and the other was ordering ( 3 problems). Each of the 3 ordering problems covered a specific component of the alpha receptor pathway and was considered to be of equal difficulty by the two physiology professors who developed the problems. Two postdoctoral physiology researchers and two graduate students reviewed and solved the problems independently before the experiment. As their answers were in $100 \%$ agreement, they were used as the answer key to grade the participants' responses.

In this paper, we report the analyses and the results for the ordering problems. Each of the ordering problems included 6 to 7 sub-steps of the physiologic pathway that the participants had learned about by watching either the media containing the dynamic animations or static images. The participants were randomly assigned a media type before he/she began the study. In the instructions for each ordering problem, the participants were tasked with putting the steps in the correct order (Figure 2). The websites on which the problems were presented allowed the participants to move their choices from the right side of the screen to ordered step boxes on the left side (i.e., drag-and-drop). If a choice had already been moved to a specific step box, it could be moved to a new step box. In either case, when a participant moved a choice into a step box, the initial location of that choice, either above the line on the right side or in the box on the left side, became empty. If the participant dragged a choice out of a step box and released the mouse without moving the choice into a new step box, the choice automatically returned to its original location.

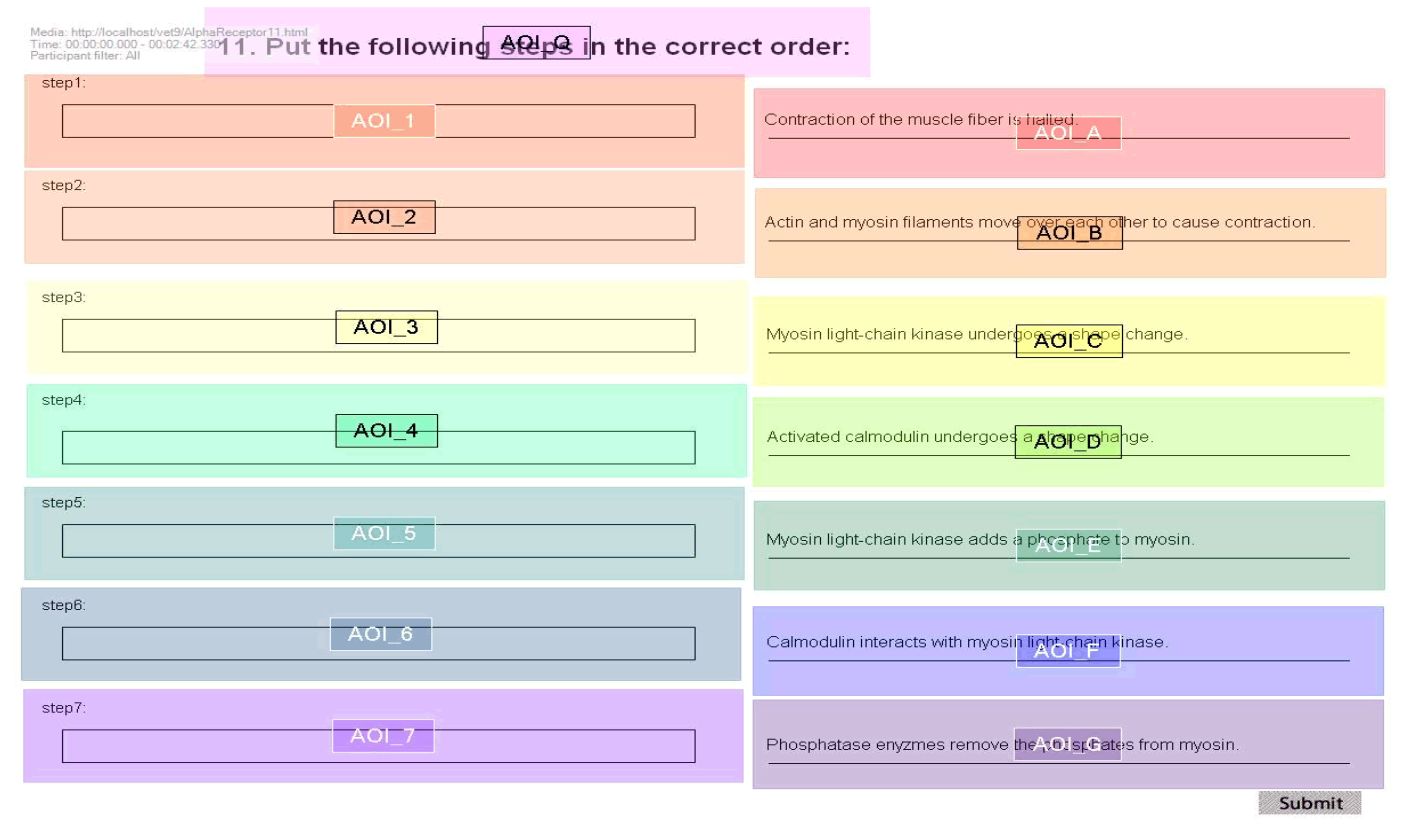

Figure 2. Superimposition of area of interest (AOI) fields on the screen capture of the third ordering problem. AOIs 1 through 7 are for the steps (left side), AOIs A through G are for the choices (right side), and AOI_Q is for question stem. 


\section{Procedure}

After reading the instructions and signing the consent form, the participants sat in front of the eye tracker screen at a distance of $60-65 \mathrm{~cm}$. They were required to sit as still as possible during the experiment to minimize gaze drift. Once the eye tracker was calibrated, participants watched their assigned media twice. After the instructor stopped the eye tracker, the participants were asked to relax for 1 minute before the eye tracker was recalibrated. Finally, the participants were instructed to solve the problems at their own paces. The experiment lasted 30 to 50 minutes, depending on the participants.

\section{Data Analysis}

AOIs were defined for each choice, step box, and the problem stem: letters for choices/problem stem and digits for steps (Figure 2). The first approach used for data analysis in this study involved using logistic regressions to explore relationships between how well the participants solved the problems and a set of independent variables. The dependent variable was the participants' total scores earned for solving the three problems. A student earned 1 point if the order of a problem was completely correct; otherwise, 0 points were assigned. Thus, a participant's total score could be $0,1,2$, or 3 for all the three problems. We also used another grading system in which the grade was based on the Levenshtein distance (Tang et al., 2012) between a participant's answer and the answer key. Levenshtein distance is used to quantitatively compare differences between two strings such as scanpaths (Tang et al., 2012; Feusner \& Lukoff, 2008). Because the answers of ordering problems are in the form of strings, this grading system is rational to assign partial credit if part of the step order in a problem is placed correctly (although we have not found literature using Levenshtein distance for grading purpose). For example, the answer key of Problem 3 is DFCEBGA (Figure 5). If a participant placed the order as DFCEB $\underline{A G}$, the Levenshtein distance was 2 and the grade was $7-2=5$.

The independent variables in the logistic regressions included media type, total fixation duration (sum of all the fixation durations within an AOI or AOI groups), fixation count, visit count, time on task and number of mouse clicks (hereafter mouse clicks). In the analysis, fixations on each problem (i.e., sum of AOIs) rather than on each AOI were examined. However, there were still 18 independent variables for the three problems. Based on previous studies, it was likely that some of these variables could be correlated, particularly the eye movement measures (Stieff \& Hegarty, 2011; Tang \& Pienta, 2012; Tang et al., 2014; Williamson et al., 2013; Jang et al., 2011). Therefore, we explored the correlations between these variables and expected the dimension of data to be reduced based on the results. All the analyses were performed using R 3.1.2 (R Core Team, 2015).

The second approach used for data analysis was a comparison of the eye movement patterns between the participants who solved the problems correctly ("correct group") and those who solved them incorrectly ("incorrect group"). This analysis was conducted using a software tool developed by our research team, which had the similar functions of eyePatterns (West et al., 2006) and was written in R. In the software tool, a scanpath pattern is a subsequence with at least three AOIs that presents in a certain number of the scanpaths. In this study, we used "collapsed" scanpaths and $20 \%$ was used as the cut-off to define a scanpath pattern. That is, a subsequence with at least three AOIs that was shared by at least $20 \%$ of the scanpaths in a group was considered to be a pattern. For example, if there are 30 scanpaths in a group and a subsequence with three AOIs can be detected in six of the scanpaths, then this subsequence is a pattern of this group. If no pattern was identified in a group using the $20 \%$ cutoff, a lower cutoff (e.g., $15 \%$ or $10 \%$ ) was employed. A group of scanpaths usually has multiple patterns and each scanpath in the group may contain none, one or more than one pattern.

To further understand the eye movement patterns in different groups and phases, we also analyzed fixational transitions within the choices (e.g., 21 and 35), within the steps (e.g., BA and CF), and between these two (e.g., 5G and E4). The sum of the numbers of within-choices and within-steps transitions relative to the number of between transitions in each phase is defined as the ratio of fixational transitions in the phase, which quantifies scanpath patterns to an extent.

\section{Results}

\section{Problems}

The numbers (\%) of participants who solved problems 1,2 and 3 correctly were $46(56.8 \%), 9$ (11.1\%) and $47(58.0 \%)$, respectively. All of the correlations (Pearson's $r$ ) between the variables (total 
fixation duration, fixation count, visit count, time on task and mouse clicks) within the same problem were larger than 0.56, indicating large effect sizes according to Cohen's criteria (Cohen, 1988). Specifically, total fixation duration, fixation count and time on task were highly correlated ( $r \geq 0.95$ ). The correlation matrix is presented in the Appendix. As a result, total fixation duration was used for the subsequent analyses because it is one of the most commonly-reported eye movement measures and it could represent the other variables. The average total fixation durations for students who solved each problem correctly and incorrectly are shown in Figure 3.

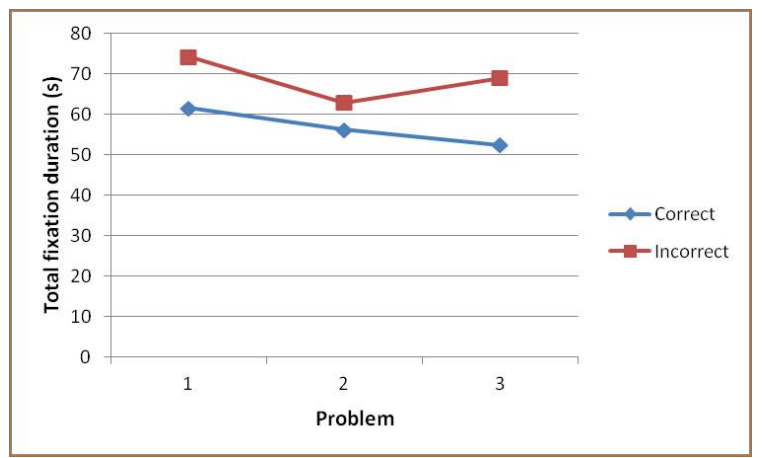

Figure 3. Average total fixation durations in each problem.

Results of a logistic regression performed to explore the relationship between the total score and total fixation duration in each problem as well as media type are presented in Table 1. An ordered logistic regression was performed rather than a binomial logistic regression because the dependent variable (score) had more than two possible values (Venables \& Ripley, 2002): 0, 1, 2, and 3 (see Data Analysis).

Table 1

Logistic regression results showing relationship between students' scores and total fixation duration in each problem as well as media type.

\begin{tabular}{lcccc}
\hline & Coefficient & Std. Error & t value & p value \\
\cline { 2 - 5 } Media Type & 0.161 & 0.424 & 0.379 & 0.705 \\
Problem 1 & -0.017 & 0.008 & -2.026 & $0.043^{*}$ \\
Problem 2 & 0.013 & 0.011 & 1.233 & 0.217 \\
Problem 3 & -0.023 & 0.010 & -2.266 & $0.023^{*}$ \\
\hline$* p<0.05$ & & & &
\end{tabular}

The total fixation durations in Problems 1 and 3 between the correct and incorrect groups were significantly different $(p<0.05)$. The negative coefficients indicated that participants with lower scores fixated longer on the problem. The total fixation durations in Problem 2 between the two groups were not significantly different, nor were media types. Because of the strong correlations identified previously between the five measures, the findings for total fixation duration should apply equally to fixation count, visit count, time on task, and mouse clicks.

\section{Phases}

The average total fixation duration in each problemsolving phase and the percentage of each phase in a problem are listed in Table 2. The total fixation durations in Phase 1 for each problem had a narrow range (17.40s $\pm 0.45 \mathrm{~s})$, as did the percentages of Phase 2 durations $(62.32 \% \pm 1.07 \%)$. Because nine independent variables ( 3 phases $\times 3$ problems) and media type remained, dimension reduction was again considered before the logistic regression was conducted.

\section{Table 2}

Average total fixation duration (in seconds) in each phase and the percentage in a problem.

\begin{tabular}{|c|c|c|c|c|c|}
\hline Problem & Group & Phase 1 & Phase 2 & Phase 3 & Sum \\
\hline \multirow{3}{*}{1} & incorrect & $\begin{array}{c}18.51 \\
(24.92 \%)\end{array}$ & $\begin{array}{c}50.38 \\
(67.82 \%)\end{array}$ & $\begin{array}{c}5.39 \\
(7.26 \%)\end{array}$ & 74.29 \\
\hline & correct & $\begin{array}{c}15.77 \\
(25.62 \%)\end{array}$ & $\begin{array}{c}37.87 \\
(61.54 \%)\end{array}$ & $\begin{array}{c}7.90 \\
(12.84 \%)\end{array}$ & 61.54 \\
\hline & average & $\begin{array}{c}16.95 \\
(25.83 \%)\end{array}$ & $\begin{array}{c}43.28 \\
(63.39 \%)\end{array}$ & $\begin{array}{c}6.82 \\
(10.78 \%)\end{array}$ & $\begin{array}{c}67.04 \\
(100 \%)\end{array}$ \\
\hline \multirow{3}{*}{2} & incorrect & $\begin{array}{c}17.64 \\
(28.06 \%)\end{array}$ & $\begin{array}{c}39.82 \\
(63.32 \%)\end{array}$ & $\begin{array}{c}5.42 \\
(8.62 \%)\end{array}$ & 62.89 \\
\hline & correct & $\begin{array}{c}19.45 \\
(34.62 \%)\end{array}$ & $\begin{array}{c}33.04 \\
(58.80 \%)\end{array}$ & $\begin{array}{c}3.70 \\
(6.58 \%)\end{array}$ & 56.18 \\
\hline & average & $\begin{array}{c}17.84 \\
(31.14 \%)\end{array}$ & $\begin{array}{c}39.07 \\
(61.25 \%)\end{array}$ & $\begin{array}{c}5.23 \\
(7.61 \%)\end{array}$ & $\begin{array}{c}62.14 \\
(100 \%)\end{array}$ \\
\hline \multirow{3}{*}{3} & incorrect & $\begin{array}{c}17.25 \\
(25.00 \%)\end{array}$ & $\begin{array}{c}49.34 \\
(71.53 \%)\end{array}$ & $\begin{array}{c}2.39 \\
(3.47 \%)\end{array}$ & 68.98 \\
\hline & correct & $\begin{array}{c}17.59 \\
(33.51 \%)\end{array}$ & $\begin{array}{c}31.63 \\
(60.27 \%)\end{array}$ & $\begin{array}{c}3.26 \\
(6.22 \%)\end{array}$ & 52.49 \\
\hline & average & $\begin{array}{c}17.44 \\
(31.80 \%)\end{array}$ & $\begin{array}{c}39.07 \\
(63.12 \%)\end{array}$ & $\begin{array}{c}2.90 \\
(5.08 \%)\end{array}$ & $\begin{array}{c}59.41 \\
(100 \%)\end{array}$ \\
\hline
\end{tabular}

This confirms the findings in Table 1 that in general, total fixation duration in each phase was not dependent on number of steps in problem. Therefore, the average total fixation duration of the three problems in each phase was included in the subsequent logistic 
regression.

The results of the ordered logistic regression, presented in Table 3, indicate that as a whole, participants with lower scores fixated significantly longer in Phase 2, whereas participants with higher scores fixated significantly longer in Phase 3.

Table 3

Logistic regression results showing relationship between student score and total fixation duration in each phase.

\begin{tabular}{lcccc}
\hline & Coefficient & Std. Error & t value & p value \\
\cline { 2 - 5 } $\begin{array}{l}\text { Media } \\
\text { Type }\end{array}$ & 0.024 & 0.433 & 0.055 & 0.956 \\
Phase1 & 0.000 & 0.030 & -0.012 & 0.991 \\
Phase2 & -0.061 & 0.015 & -3.999 & $0.000 * *$ \\
Phase3 & 0.097 & 0.047 & 2.050 & $0.040^{*}$ \\
\hline$* p<0.05$ & & & &
\end{tabular}

Using the Levenshtein distance system in which partial credits were assigned to the part of the step order that was placed correctly, the results were similar to those obtained (Tables 1 and 3) when 1 point was assigned only if the step order in a problem was completely correct.

\section{Scanpath patterns}

As an example, the scanpaths (gaze plots) for Problem 3 from two participants (one correct and one incorrect, in green and purple, respectively) are shown in Figure 4. The difference of gaze plots on the step side can be observed: for the correct participant, the fixation counts generally decrease from step 1 to step 7; for the incorrect participant, gazes were roughly distributed on each step. To illustrate the details of scanpaths, the patterns in each phase of students who solved Problem 3 correctly and incorrectly were listed side by side (Figure 5 ).

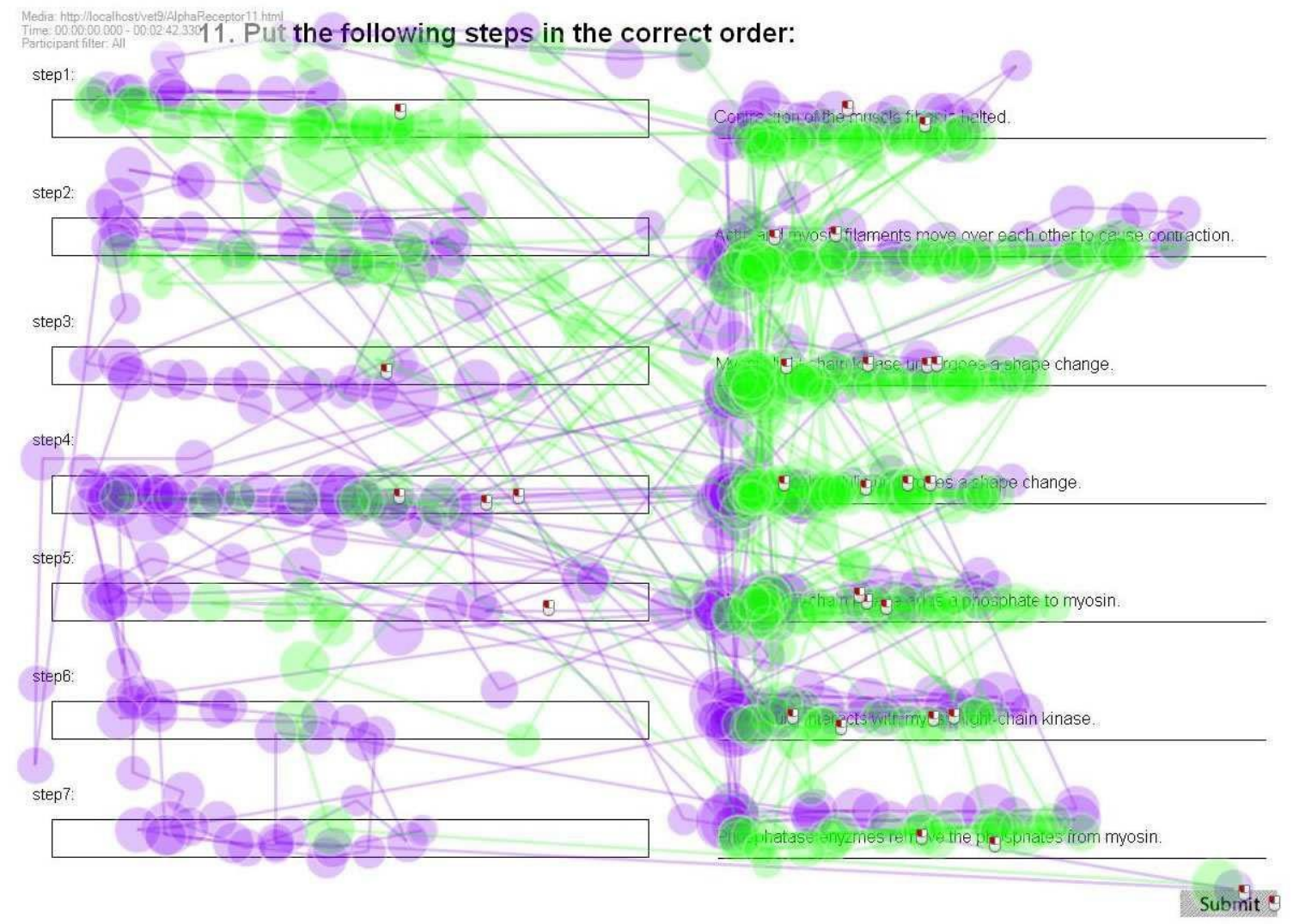

Figure 4. Gaze plots of the third ordering problem from a student who solved the problem correctly (green) and student who solved incorrectly (purple). 


\begin{tabular}{|c|c|c|c|}
\hline \multicolumn{2}{|c|}{ Correct } & \multicolumn{2}{|c|}{ Incorrect } \\
\hline patterns & percent & patterns & percent \\
\hline ABCDEFG & $29.79 \%$ & BCDEFG & $29.41 \%$ \\
\hline ABCDEF & $31.91 \%$ & $\mathrm{ABCDEF}$ & $26.47 \%$ \\
\hline BCDEFG & $31.91 \%$ & $\mathrm{BCDEF}$ & $41.18 \%$ \\
\hline$A B C D E$ & $40.43 \%$ & CDEFG & $41.18 \%$ \\
\hline $\mathrm{BCDEF}$ & $36.17 \%$ & $A B C D E$ & $38.24 \%$ \\
\hline CDEFG & $31.91 \%$ & QABCD & $20.59 \%$ \\
\hline $\mathrm{BCDE}$ & $51.06 \%$ & $\mathrm{BCDE}$ & $55.88 \%$ \\
\hline$A B C D$ & $44.68 \%$ & $\mathrm{CDEF}$ & $52.94 \%$ \\
\hline DEFG & $42.55 \%$ & $\mathrm{ABCD}$ & $50.00 \%$ \\
\hline CDEF & $40.43 \%$ & DEFG & $50.00 \%$ \\
\hline QABC & $27.66 \%$ & QABC & $29.41 \%$ \\
\hline $1 \mathrm{ABC}$ & $23.40 \%$ & $1 \mathrm{ABC}$ & $23.53 \%$ \\
\hline EFGF & $23.40 \%$ & $\mathrm{BCBC}$ & $20.59 \%$ \\
\hline BABC & $21.28 \%$ & $\mathrm{ABC}$ & $73.53 \%$ \\
\hline$A B C$ & $63.83 \%$ & $\mathrm{BCD}$ & $73.53 \%$ \\
\hline $\mathrm{CDE}$ & $57.45 \%$ & $\mathrm{CDE}$ & $67.65 \%$ \\
\hline $\mathrm{BCD}$ & $57.45 \%$ & DEF & $64.71 \%$ \\
\hline EFG & $55.32 \%$ & EFG & $58.82 \%$ \\
\hline DEF & $53.19 \%$ & QAB & $35.29 \%$ \\
\hline EDC & $36.17 \%$ & $1 \mathrm{AB}$ & $29.41 \%$ \\
\hline QAB & $31.91 \%$ & BAB & $26.47 \%$ \\
\hline $\begin{array}{l}\text { FGF } \\
\text { BAB }\end{array}$ & $\begin{array}{l}29.79 \% \\
27.66 \%\end{array}$ & ABA & $26.47 \%$ \\
\hline $\begin{array}{l}\text { BAB } \\
\text { GFE }\end{array}$ & $\begin{array}{l}27.66 \% \\
27.66 \%\end{array}$ & CBC & $26.47 \%$ \\
\hline FED & $27.66 \%$ & FGF & $26.47 \%$ \\
\hline $1 \mathrm{AB}$ & $25.53 \%$ & 1QA & $20.59 \%$ \\
\hline DED & $23.40 \%$ & $A Q A$ & $20.59 \%$ \\
\hline$D C D$ & $23.40 \%$ & $\mathrm{BCB}$ & $20.59 \%$ \\
\hline 1QA & $23.40 \%$ & & \\
\hline EDE & $21.28 \%$ & & \\
\hline
\end{tabular}

\begin{tabular}{|c|c|c|c|}
\hline \multicolumn{2}{|c|}{ Correct } & \multicolumn{2}{|c|}{ Incorrect } \\
\hline patterns & percent & patterns & percent \\
\hline 212 & $36.17 \%$ & 212 & $35.29 \%$ \\
\hline BAB & $31.91 \%$ & $3 \mathrm{C} 3$ & $32.35 \%$ \\
\hline G6A & $29.79 \%$ & 323 & $29.41 \%$ \\
\hline $5 \mathrm{G} 6$ & $27.66 \%$ & CDE & $29.41 \%$ \\
\hline 4BA & $25.53 \%$ & CBC & $26.47 \%$ \\
\hline $\mathrm{F} 21$ & $23.40 \%$ & $\mathrm{BAB}$ & $23.53 \%$ \\
\hline $2 \mathrm{C} 3$ & $23.40 \%$ & 545 & $23.53 \%$ \\
\hline \multirow[t]{7}{*}{ D21 } & $21.28 \%$ & 434 & $20.59 \%$ \\
\hline & & 234 & $20.59 \%$ \\
\hline & & 454 & $20.59 \%$ \\
\hline & & 543 & $20.59 \%$ \\
\hline & & $21 \mathrm{~A}$ & $20.59 \%$ \\
\hline & & $23 \mathrm{D}$ & $20.59 \%$ \\
\hline & & $B C D$ & $20.59 \%$ \\
\hline \multicolumn{2}{|c|}{ answer: } & C3C & $20.59 \%$ \\
\hline \multicolumn{2}{|c|}{ D-1 } & & \\
\hline \multicolumn{2}{|c|}{$\mathrm{F}-2$} & & \\
\hline \multicolumn{2}{|c|}{ C-3 } & & \\
\hline \multicolumn{2}{|c|}{ E-4 } & & \\
\hline \multicolumn{2}{|c|}{ B-5 } & & \\
\hline \multicolumn{2}{|c|}{ G-6 } & & \\
\hline \multicolumn{2}{|c|}{ A-7 } & & \\
\hline
\end{tabular}

\begin{tabular}{lll}
\multicolumn{2}{c}{ Phase3 $(\geq 10 \%)$} \\
Correct & Incorrect \\
& & \\
patterns & percent & No \\
1234 & $10.64 \%$ & data \\
3456 & $10.64 \%$ & \\
4567 & $10.64 \%$ & \\
456 & $19.15 \%$ & \\
345 & $17.02 \%$ & \\
234 & $14.89 \%$ & \\
123 & $14.89 \%$ & \\
567 & $14.89 \%$ & \\
$67 \mathrm{G}$ & $12.77 \%$ &
\end{tabular}

Figure 5. Scanpath patterns in each phase of the third ordering problem for students who solved the problem correctly and incorrectly. For each group (correct or incorrect), column 1 contains the scanpath pattern and column 2 contains the percentage of the occurrence of pattern, i.e., the ratio of the number of participant who had a pattern to the total number of participants in a group. The definition of AOIs is listed at the bottom of Phase 3, and the correct order of the choices (answer key) in Problem 3 is listed at the bottom of Phase 2. For example, choice D should be placed in step box 1, choice F in step box 2, choice $C$ in step box 3 , etc.

As is evident in Phase 1 (Figure 5), the correct and incorrect groups had similar scanpath patterns. Their eye movements were mostly between adjacent choices, e.g., DCD or FED, without any jumps (e.g., no patterns such as ADE were identified). There were also a few patterns involving the problem stem (e.g., QABCD) and the first step (e.g., $1 \mathrm{ABC}$ ).

In Phase 2, the correct and incorrect groups had different scanpath patterns. While the eye movements in the incorrect group were still between adjacent choices and/or steps such as 545 and $21 \mathrm{~A}$, most patterns in the correct group revealed eye movements between choices and the corresponding correct steps such as F21 and 5G6 (both F-2 and G-6 are correct choice-step connections).

In Phase 3, neither group had patterns shared by at least $20 \%$ of the participants. When $10 \%$ was used as the cutoff value, the scanpath patterns in the correct group revealed that participants checked their answers step-by-step. There was no scanpath pattern identified in the incorrect group, suggesting that participants who solved the problem incorrectly either did not check their answers or their eye fixations were largely scattered if they performed any "answer-checking." The latter is supported by the previous finding that the participants who ordered the steps in a problem correctly fixated longer on Phase 3 of the problem, than the participants who ordered the steps incorrectly.

The average numbers of within-choices, withinsteps and between fixational transitions in each phase of each problem in different groups, as well as the ratios are shown in Figure 6. The characteristics of fixational transitions in the same phase were consistent among the three problems. In Phase 1, most fixational transitions were within-choices. There were some between-transitions, but very few within-steps ones. In Phase 2, there were relatively more between-transitions than within ones. In Phase 3 , most transitions were within-steps. There were some between-transitions ("mix"), but very few within-choices ones. The ratios in Phase 2 were around 1, and the ratios in the other two 
phases were much larger. Furthermore, in general, the incorrect group had larger within-to-between ratios.

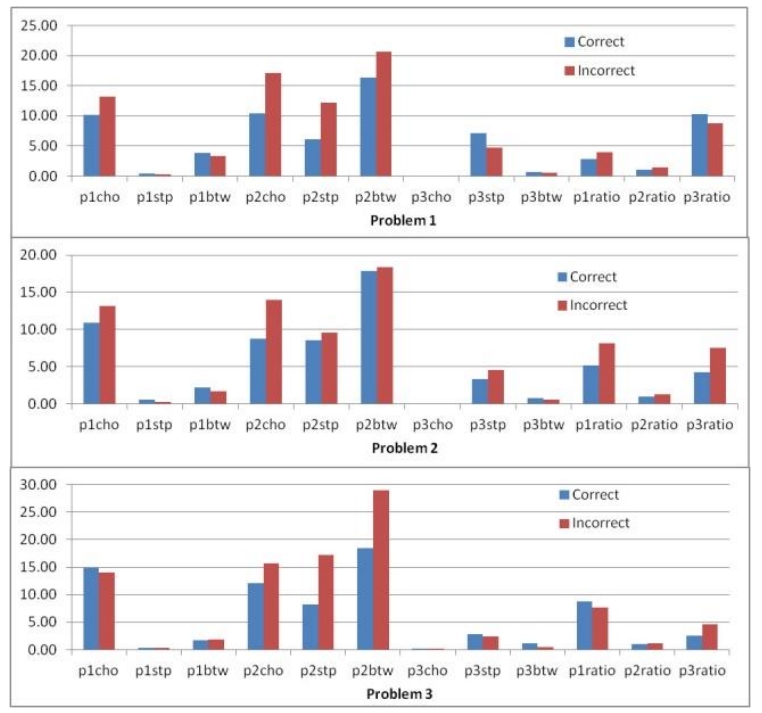

Figure 6. Average numbers and ratios of fixational transitions in each phase of each problem and each group. $p=$ phase, cho=within-choices, st $p=$ within-steps, btw $=$ between.

\section{Discussion}

The results of the present study revealed that students with higher scores fixated for a significantly shorter amount of time on the choice in the problemsolving phase (Phase 2), but fixated longer in the answer-checking phase (Phase 3). We also used another grading system in which a student was assigned partial credits if part of the step order in a problem was placed correctly. The results were similar to those where a student was assigned 1 point only if the step order in a problem was completely correct. The differences in total fixation duration between the correct and incorrect groups can be explained by the results obtained after comparing the scanpath patterns (Figure 5). In Phase 2, most eye movement patterns in the correct group were connections between choices and the corresponding correct step boxes, which was evidence of an efficient problem-solving strategy. In contrast, the eye fixations of participants in the incorrect group wandered more across adjacent choices and/or step boxes (e.g., 545 and $21 \mathrm{~A}$ ). This difference was supported by the results that in Phase 2, the within-to-between ratios of fixational transitions in the incorrect group were larger than those in the correct group (Figure 6). In Phase 3, participants in the correct group checked their answers in order, whereas there was no evidence that participants in the incorrect group checked their answers, although the majority of the fixational transitions in the incorrect group also were within-steps (Figure 6). The results showed that answer-checking was more likely an indicator of better outcome/performance in problemsolving or a test.

In Phase 1, there was no significant difference in total fixation duration between the two groups, and the scanpath analysis revealed that both groups had very similar eye movement patterns (i.e., primarily between neighboring choices, e.g., DCD or FED). Thus, Phase 1 was actually a "reading" stage, which was also supported by the scanpath patterns that revealed normal reading orders (e.g., $\mathrm{QABCD}$ and $\underline{\mathrm{ABC}}$, see Figure 5), and by the findings that most fixational transitions in this phase were within-choices (Figure 6). In other words, before completely moving a choice into a step box, none of the participants formulated a "plan" that could be detected by eye-tracking. Therefore, it appears that "planning" was integrated with "problem-solving" in Phase 2. As summarized in Table 2, the total fixation durations in Phase 1 on different choices were not different for participants in the two groups. This finding is consistent with those indentified in our previous studies regarding the reading phase (Tang \& Pienta, 2012; Tang et al., 2014). Thus, if students are at a similar academic level (e.g., enrolled in the same course), their total fixation durations in the reading phase are relatively constant when they solve a science problem. The durations may only vary according to the length of problem, regardless of whether they ultimately solve the problem correctly or not.

There is evidence in the literature that dynamic animations are more effective in aiding student learning than static images (Buchanan et al, 2005; Tversky, Morrison \& Betrancourt, 2002; Aldahmash \& Abraham, 2009). However, the results of the logistic regressions in this study indicated that media type did not positively impact students' scores. There are two explanations for why no significant difference was discovered, with the first being that some students might have had prior knowledge in this topic area. Approximately $80 \%$ of the participants had previously taken a biochemistry course in which the pathway used in this study could have been covered. If most of the participants had prior knowledge in this content area, we would not expect to see a large difference due to media type. The fact that $>88 \%$ of the students answered Problem 2 incorrectly would suggest that prior exposure to the pathway did not, however, equate with a full understanding of the materials. The second reason for 
the lack of a difference due to media type could be that the static images themselves were taken from the dynamic animations and were quite detailed. Given how comprehensive these static images were, it is possible there was not enough difference between the static images and the dynamic animations to discover a significant difference between the participants who watched each media type.

In this study, we found that total fixation duration, fixation count, visit count, time on task and mouse clicks were positively correlated with each other. This result provides additional reliable evidence supporting similar findings regarding fixation duration and fixation count detected in our previous research studies (Tang \& Pienta, 2012; Tang et al., 2014) and by others in studies on problem-solving (Stieff \& Hegarty, 2011; Williamson et al., 2013; Jang et al., 2011). Because studies addressing this issue included different problem formats (e.g., multiple-choice, word problems and ordering problems) and diverse disciplines in science education (e.g., chemistry and physiology), it appears that at least in science problem-solving research, (total) fixation duration and fixation count are interchangeable. In many eye-tracking studies, researchers have reported multiple eye movement measures simultaneously. For example, in the fields of psychology and reading, where more than one experiment usually is included in a study, fixation duration and fixation count are often analyzed and reported in each of the experiments, though the results on these two measures could be very similar. The results from the present study suggest that correlations between different eye movement measures should be examined to avoid repetitive analyses.

Moreover, researchers have used eye-tracking data to predict viewing (Kanan, Ray, Bseiso, Hsiao \& Cottrell, 2014; Greene, Liu \& Wolfe, 2012) or problem-solving behaviors and outcomes (Bednarik, Eivazi \& Vrzakova, 2013; French \& Thibaut, 2014; Eivazi \& Bednarik, 2011; Tsai, Viirre, Strychacz, Chase \& Jung, 2007). In most predicting models, multiple eye movement measures are selected as predictor variables. These measures include fixation duration, number of fixations, fixation angles and saccade amplitude. According to the findings in the literature and the present study, some of these measures may be highly correlated. Therefore, researchers are advised to consider collinearity when building predictive models. If some eye movement measures are highly correlated, it is appropriate to remove variables from the models (Dormann et al., 2013).

\section{Limitations and Future Work}

In Phase 2, the scanpaths were generated directly from the eye-tracking data. Thus, an AOI represented eye fixations on the original location of a choice or step box. For example, when $\mathrm{AOI}=\mathrm{A}$, the participants could be looking at the location of the first choice, regardless of whether the choice was still present or had been moved (although the possibility that latter scenario happened was very low). Our concern would be similar for $\mathrm{AOI}=1$, for which our scanpath data did not inform us as to which choice was in the first step box where the participants were fixating; or whether the participants were fixating on an empty step box. Thus, although the scanpath patterns in this phase revealed logic connections between choices and the corresponding correct steps (in the correct group), combining both eye-tracking and action recordings should provide more accurate information. Moreover, in the present study, eye movement patterns were compared mainly between two groups of participants. In the future, we also plan to compare more withinsubject behaviors. For example, if a participant solves one problem correctly and solves another one incorrectly, are there differences in eye movement patterns between the two problem-solving processes?

\section{Conclusions}

The key finding of the current study was that the total fixation duration of students with higher scores was significantly shorter on 2 of the 3 problems than that of students with lower scores. By analyzing data for each phase of the problem-solving process, significant differences were detected during Phase 2, i.e., planning and problem-solving, which is consistent with the findings in our previous studies (Tang \& Pienta, 2012), as well as during Phase 3, the answerchecking phase. In addition, the scanpath analysis revealed different eye movement patterns in these two phases of problem-solving between correct and incorrect groups of participants. These results indicate that ordering problems are appropriate types of activities for eye-tracking researchers to explore problem-solving strategies. Finally, no significant differences in eye movement measures were identified between students who watched the media featuring dynamic animations and static images. 


\section{References}

Aldahmash, A. H., \& Abraham, M. R. (2009). Kinetic versus static visuals for facilitating college students' understanding of organic reaction mechanisms in chemistry. Journal of Chemical Education, 86, 1442-1446.

Bednarik, R., Eivazi, S., \& Vrzakova, H. (2013). A Computational Approach for Prediction of ProblemSolving Behavior Using Support Vector Machines and Eye-Tracking Data. In Eye Gaze in Intelligent User Interfaces (pp. 111-134). Springer London.

Buchanan, M. F., Carter, W. C., Cowgill, L. M., Hurley, D. J., Lewis, S. J., MacLeod, J. N., \& Robertson, T. P. (2005). Using 3D animations to teach intracellular signal transduction mechanisms: taking the arrows out of cells. Journal of veterinary medical education, 32(1), 72-78.

Chi, M. T. (1997). Quantifying qualitative analyses of verbal data: A practical guide. The journal of the learning sciences, 6(3), 271-315.

Cohen, J. (1988). Statistical power analysis for the behavioral sciences (2nd ed.). Hillsdale, NJ: Lawrence Earlbaum Associates.

De Corte, E., Verschaffel, L., \& Pauwels, A. (1990). Influence of the semantic structure of word problems on second graders' eye movements. Journal of Educational Psychology, 82(2), 359.

Doherty, S., O’Brien, S., \& Carl, M. (2010). Eye tracking as an MT evaluation technique. Machine translation, 24(1), 1-13.

Dormann, C. F., Elith, J., Bacher, S., Buchmann, C., Carl, G., Carré, G., \& Münkemüller, T. (2013). Collinearity: a review of methods to deal with it and a simulation study evaluating their performance. Ecography, 36(1), 27-46.

Eivazi, S., \& Bednarik, R. (2011). Predicting problemsolving behavior and performance levels from visual attention data. In Proc. Workshop on Eye Gaze in Intelligent Human Machine Interaction at IUI (pp. 9-16).

Epelboim, J., \& Suppes, P. (2001). A model of eye movements and visual working memory during problem solving in geometry. Vision Research, 41(12), 1561-1574.

French, R. M., \& Thibaut, J. P. Using eye-tracking to predict children's success or failure on analogy tasks. In Livre/Conférence Proceedings of the Thirty-Sixth Annual Meeting of the Cognitive
Science Society. Austin, TX: Cognitive Science Society (pp. 2222-2227).

Feusner, M., \& Lukoff, B. (2008, March). Testing for statistically significant differences between groups of scan patterns. In Proceedings of the 2008 symposium on Eye tracking research \& applications (pp. 43-46). ACM.

Grant, E. R., \& Spivey, M. J. (2003). Eye movements and problem solving guiding attention guides thought. Psychological Science, 14(5), 462-466.

Green, H. J., Lemaire, P., \& Dufau, S. (2007). Eye movement correlates of younger and older adults' strategies for complex addition. Acta psychologica, 125(3), 257-278.

Greene, M. R., Liu, T., \& Wolfe, J. M. (2012). Reconsidering Yarbus: A failure to predict observers' task from eye movement patterns. Vision research, 62, 1-8.

Hegarty, M., Mayer, R. E., \& Green, C. E. (1992). Comprehension of arithmetic word problems: Evidence from students' eye fixations. Journal of Educational Psychology, 84(1), 76.

Hertzum, M., Hansen, K. D., \& Andersen, H. H. (2009). Scrutinising usability evaluation: does thinking aloud affect behaviour and mental workload? Behaviour \& Information Technology, 28(2), 165-181.

Holmqvist, K., Nyström, M., Andersson, R., Dewhurst, R., Jarodzka, H., \& Van de Weijer, J. (2011). Eye tracking: A comprehensive guide to methods and measures. Oxford University Press. Chapter 9.

Jang, Y. M., Lee, S., Mallipeddi, R., Kwak, H. W., \& Lee, M. (2011, January). Recognition of human's implicit intention based on an eyeball movement pattern analysis. In Neural Information Processing (pp. 138-145). Springer Berlin Heidelberg.

Just, M. A, \& Carpenter, P. A. (1984). Using eye fixations to study reading compre hension. In D. E. Kieras \& M. A. Just (Eds.), New Methods in Reading Comprehension Research (pp. 151-182). Hillsdale, NJ: Erlbaum.

Kanan, C., Ray, N. A., Bseiso, D. N., Hsiao, J. H., \& Cottrell, G. W. (2014, March). Predicting an observer's task using multi-fixation pattern analysis. In Proceedings of the symposium on eye tracking research and applications (pp. 287-290). ACM.

Kruger, J., Hefer, E., \& Matthew, G. (2014). Attention distribution and cognitive load in a subtitled 
academic lecture: L1 vs. L2 Journal of Eye Movement Research, 7(5), 4.

Levenshtein, V. I. (1966). Binary codes capable of correcting deletions, insertions, and reversals. In Soviet physics doklady, 10(8), 707-710.

Mayer, R. E., Larkin, J. H., \& Kadane, J. B. (1984). A cognitive analysis of mathematical problem-solving ability. In R. J. Sternberg (Ed.), Advances in the psychology of human intelligence (Vol. 2, pp. 231273). Hillsdale, NJ: Erlbaum.

Peebles, D., \& Cheng, P. C. H. (2001). Graph-based reasoning: From task analysis to cognitive explanation. In Proceedings of the twenty-third annual conference of the cognitive science society (pp. 762-767).

Poole, A., \& Ball, L. J. (2006). Eye tracking in HCI and usability research. Encyclopedia of human computer interaction, 1, 211-219.

R Core Team. (2015). R: A Language and Environment for Statistical Computing. $\mathrm{R}$ Foundation for Statistical Computing, Vienna, Austria. URL http://www.R-project.org/.

Reindl, K.M., White, A.R., Johnson, C., Bender, B., Slator, B.M., \& McLean, P. (2015). The virtual cell animation collection: Tools for teaching molecular and cellular biology. PLoS Biol 13(4): e1002118, $1-9$.

Slykhuis, D. A., Wiebe, E. N., \& Annetta, L. A. (2005). Eye-tracking students' attention to PowerPoint photographs in a science education setting. Journal of Science Education and Technology, 14(5-6), $509-520$.

Stieff, M.; Hegarty, M.; Deslongchamps, G. Identifying Representational Competence with Multirepresentational Displays. Cognition Instruct. 2011, 29 (1), 123-145.

Suppes, P., Cohen, M., Laddaga, R., Anliker, J., \& Floyd, R. (1983). A procedural theory of eye movements in doing arithmetic. Journal of Mathematical Psychology, 27(4), 341-369.

Tai, R. H., Loehr, J. F., \& Brigham, F. J. (2006). An exploration of the use of eye-gaze tracking to study problem-solving on standardized science assessments. International journal of research \& method in education, 29(2), 185-208.

Tang, H., \& Pienta, N. (2012). Eye-tracking study of complexity in gas law problems. Journal of Chemical Education, 89(8), 988-994.
Tang, H., Kirk, J., \& Pienta, N. J. (2014). Investigating the Effect of Complexity Factors in Stoichiometry Problems Using Logistic Regression and Eye Tracking. Journal of Chemical Education, 91(7), 969-975.

Tang, H., Topczewski, J. J., Topczewski, A. M., \& Pienta, N. J. (2012, March). Permutation test for groups of scanpaths using normalized Levenshtein distances and application in NMR questions. In Proceedings of the Symposium on Eye Tracking Research and Applications (pp. 169-172). ACM.

Tobii. (2011). Tobii Studio 2.X User Manual. Stockholm, Sweden.

Tsai, Y. F., Viirre, E., Strychacz, C., Chase, B., \& Jung, T. P. (2007). Task performance and eye activity: predicting behavior relating to cognitive workload. Aviation, space, and environmental medicine, 78(Supplement 1), B176-B185.

Tsai, M. J., Hou, H. T., Lai, M. L., Liu, W. Y., \& Yang, F. Y. (2012). Visual attention for solving multiplechoice science problem: An eye-tracking analysis. Computers \& Education, 58(1), 375-385.

Tversky, B., Morrison, J. B., \& Betrancourt, M. (2002). Animation: can it facilitate? International journal of human-computer studies, 57(4), 247-262.

van Gog, T., Paas, F., \& Van Merriënboer, J. J. G. (2005). Uncovering expertise-related differences in troubleshooting performance: Combining eye movement and concurrent verbal protocol data. Applied Cognitive Psychology, 19, 205-221.

Van Gog, T., Jarodzka, H., Scheiter, K., Gerjets, P., \& Paas, F. (2009). Attention guidance during example study via the model's eye movements. Computers in Human Behavior, 25(3), 785-791.

Venables, W. N. \& Ripley, B. D. (2002). Modern Applied Statistics with $S$ (4th ed.). Springer, New York.

West, J. M., Haake, A. R., Rozanski, E. P., \& Karn, K. S. (2006, March). eyePatterns: software for identifying patterns and similarities across fixation sequences. In Proceedings of the 2006 symposium on Eye tracking research \& applications (pp. 149154). ACM.

Williamson, V. M., Hegarty, M., Deslongchamps, G., Williamson III, K. C., \& Shultz, M. J. (2013). Identifying student use of ball-and-stick images versus electrostatic potential map images via eye tracking. Journal of Chemical Education,90(2), 159-164. 


\section{Appendix}

Table Al

Correlation matrix of fixation duration, fixation count, visit count, time on task and mouse click in each of the three problems

\begin{tabular}{|c|c|c|c|c|c|c|c|c|c|c|c|c|c|c|c|c|}
\hline \multirow{4}{*}{ Fixation } & \multirow[b]{2}{*}{ blem } & \multicolumn{3}{|c|}{$\begin{array}{l}\text { Fixation } \\
\text { Duration }\end{array}$} & \multicolumn{3}{|c|}{ Fixation Count } & \multicolumn{3}{|c|}{ Visit Count } & \multicolumn{3}{|c|}{ Time on Task } & \multicolumn{3}{|c|}{ Mouse Clicks } \\
\hline & & 1 & 2 & 3 & 1 & 2 & 3 & 1 & 2 & 3 & 1 & 2 & 3 & 1 & 2 & 3 \\
\hline & 1 & & 0.308 & 0.399 & 0.977 & 0.308 & 0.411 & 0.856 & 0.141 & 0.331 & 0.990 & 0.302 & 0.406 & 0.663 & -0.003 & 0.153 \\
\hline & 2 & & & 0.430 & 0.263 & 0.968 & 0.441 & 0.081 & 0.766 & 0.352 & 0.272 & 0.973 & 0.382 & -0.089 & 0.606 & 0.185 \\
\hline Duration & 3 & & & & 0.344 & 0.389 & 0.974 & 0.240 & 0.274 & 0.878 & 0.377 & 0.425 & 0.976 & 0.095 & 0.138 & 0.713 \\
\hline Fixation & 1 & & & & & 0.303 & 0.389 & 0.896 & 0.149 & 0.316 & 0.976 & 0.264 & 0.355 & 0.677 & -0.033 & 0.129 \\
\hline & 2 & & & & & & 0.445 & 0.125 & 0.809 & 0.359 & 0.277 & 0.949 & 0.342 & -0.083 & 0.566 & 0.154 \\
\hline Count & 3 & & & & & & & 0.280 & 0.332 & 0.903 & 0.393 & 0.443 & 0.950 & 0.118 & 0.156 & 0.699 \\
\hline Visit & 1 & & & & & & & & 0.147 & 0.325 & 0.891 & 0.127 & 0.289 & 0.768 & -0.064 & 0.171 \\
\hline & 2 & & & & & & & & & 0.425 & 0.154 & 0.842 & 0.307 & -0.102 & 0.641 & 0.211 \\
\hline Count & 3 & & & & & & & & & & 0.330 & 0.402 & 0.915 & 0.127 & 0.206 & 0.805 \\
\hline Time & 1 & & & & & & & & & & & 0.288 & 0.400 & 0.680 & -0.016 & 0.149 \\
\hline & 2 & & & & & & & & & & & & 0.418 & -0.075 & 0.605 & 0.208 \\
\hline Spent & 3 & & & & & & & & & & & & & 0.120 & 0.124 & 0.735 \\
\hline Mouse & 1 & & & & & & & & & & & & & & -0.141 & 0.110 \\
\hline & 2 & & & & & & & & & & & & & & & 0.228 \\
\hline Click & 3 & & & & & & & & & & & & & & & \\
\hline
\end{tabular}

Red and bold: $r \geq 0.95$. Bold: $r>0.56$. 